8 Lefebvre V, Dumitriu B, Penzo-Mendez A, Han Y, Pallavi B. Control of cell fate and differentiation by Sry-related high-mobility-group box (Sox) transcription factors. Int J Biochem Cell Biol 2007; 39: 2195-2214.

9 Ek S, Dictor M, Jerkeman M, Jirstrom K, Borrebaeck CA. Nuclear expression of the non B-cell lineage Sox11 transcription factor identifies mantle cell lymphoma. Blood 2008; 111: 800-805.

10 Wang X, Asplund AC, Porwit A, Flygare J, Smith Cl, Christensson B et al. The subcellular Sox11 distribution pattern identifies subsets of mantle cell lymphoma: correlation to overall survival. $\mathrm{Br} J$ Haematol 2008; 143: 248-252.

11 Fernandez V, Salamero O, Espinet B, Sole F, Royo C, Navarro A et al. Genomic and gene expression profiling defines indolent forms of mantle cell lymphoma. Cancer Res 2010; 70: 1408-1418.
12 Nygren L, Baumgartner WS, Klimkowska M, Christensson B, Kimby E, Sander B. Prognostic role of SOX11 in a population-based cohort of mantle cell lymphoma. Blood 2012; 119: 4215-4223.

13 Ondrejka SL, Lai R, Smith SD, Hsi ED. Indolent mantle cell leukemia: a clinicopathological variant characterized by isolated lymphocytosis, interstitial bone marrow involvement, kappa light chain restriction, and good prognosis. Haematologica 2011; 96: 1121-1127.

14 Swerdlow SH, Williams ME. From centrocytic to mantle cell lymphoma: a clinicopathologic and molecular review of 3 decades. Hum Pathol 2002; 33: 7-20.

15 Bacher U, Kern W, Haferlach C, Alpermann T, Haferlach T, Schnittger S. Cyclin D1 (CCND1) mRNA expression assessed by real-time PCR is increased in patients with mantle cell lymphoma and contributes to diagnosis in B-cell lymphoma cases. Blood 2013; 120: 1595a.

\title{
OPEN
}

\section{Vertebral augmentation in the treatment of pathologic compression fractures in 792 patients with multiple myeloma}

Leukemia (2013) 27, 2391-2393; doi:10.1038/leu.2013.162

Multiple myeloma is the most common form of neoplasm affecting or involving the bone, accounts for $1 \%$ of all cancer types with a lifetime risk of $0.62 \%$, and new cases are now estimated at 21700 adults in the United States per year. ${ }^{1}$ This plasma cell dyscrasia is characterized by clonal proliferation of neoplastic plasma cells producing osteoclast-activating and osteoblast-inhibiting factors. ${ }^{2,3}$ Clinical symptoms result from pathological fractures at the site of osteolytic lesions or profound osteoporosis, often resulting in hypercalcemia. ${ }^{3}$ Symptomatic fractures are most commonly seen in the vertebral bodies centered in the thoracolumbar region. ${ }^{1,4,5}$ These types of compression fractures result in significant pain, decreased activity levels, disability and increased morbidity. ${ }^{6-8}$ We describe the fracture distribution and the efficacy of vertebral augmentation (vertebroplasty or kyphoplasty) in 792 consecutive patients with myeloma-related symptomatic compression fractures, who underwent 2693 vertebral augmentations at the University of Arkansas for Medical Sciences between January 2001 to May 2007. Information was collected on type of augmentation (vertebroplasty or kyphoplasty), patient vitals, magnetic resonance imaging, computed tomography (CT) and Positron emission tomography-CT for vertebral fracture location. All of the patients were on cancer therapy or about to receive therapy. Pre- and postprocedure pain, medication and activity level were assessed by survey in a subset of 361 subjects. When the participants were not in hospital to complete the survey, surveys were mailed to patient-reported addresses, which were often not reflective of their current living situation, as most patients (80\%) had relocated to the local area for treatment. Efficacy was assessed by changes in pain with the Visual Acuity Scale (VAS), analgesic usage and activity level pre-procedure and 1 month post procedure by chart documentation and patient survey. All patients received $1 \mathrm{~g}$ Cefazolin (Ancef) pre-procedure. Institutional Review Board approval was obtained for this study.

\section{STATISTICAL METHODS}

As many of the subjects participating in the outcomes study underwent multiple sessions, clustering of sessions within subjects was addressed by using mixed models with a random effect for subject for analyzing change in pain score, and generalized mixed models with a fixed effect for time and a random effect for subject for pain medication and physical activity.
For modeling, pain medication was dichotomized as narcotics versus all other categories and activity as no limitation or walking without assistance versus all other categories. Additional models explored age, gender, session number (first or subsequent), number of augmentations and type of repair. Outcomes were also analyzed by repair region (I-vertebra (T)T1-T10, II-thoracic (T)11-lumbar (L)2 or III-L3-sacrum) for the subset having repair(s) to a single region (excluding those with repairs $\geqslant 1$ region). To assess response bias, demographics and procedure distributions were compared between those participating in the outcomes study and those with only procedural data.

\section{RESULTS}

Of the 792 patients who underwent vertebroplasty or kyphoplasty, the median age was 63 years (range, 16-99) with 76 (10\%) patients being 80 years and older, and $56 \%$ of patients were males. These patients underwent a total of 1072 sessions; the majority of patients (75\%) had one vertebral augmentation session, $18 \%$ of patients had two vertebral augmentation sessions, $7 \%$ had three to six sessions. The number of repairs varied per session with the median being 2 (interquartile range (IQR) 1-3) repairs per session with $23 \%$ of sessions involving four to nine augmentations. The majority (83\%) of the 2693 levels treated were repaired with vertebroplasty. The distribution of vertebroplasty repairs was 37\% for T1-T10, 39\% for T11-L2 and 24\% for L3-sacrum (Figure 1a). Overall, $68 \%$ of all sessions involved repair on $\geqslant 2$ vertebra, and a similar distribution across levels was seen for patients with kyphoplasty. Two complications (0.3\%) required antibiotics. No neurological deficits were observed.

\section{Pre- and post-assessments}

For the outcomes study, 361 participants provided both pre- and post-assessments ( 1 month) for 447 sessions for at least one of the three outcomes (VAS, analgesic medications, or activity level assessments). Of these participants, $82 \%$ had assessments for one session only, $15 \%$ for two sessions and $4 \%$ for three or four sessions. Patients participating in the outcomes study were significantly more likely to be younger (median, 60 vs 65), male (63 vs $50 \%$ ) and from out of state (66 vs $36 \%$ ) than those who were non-participants (Table 1). Most patients were from out of state and relocated to the area for treatment. The number of levels repaired did not differ significantly with the median being 2 (IQR, 1-3) for both participants and non-participants. 


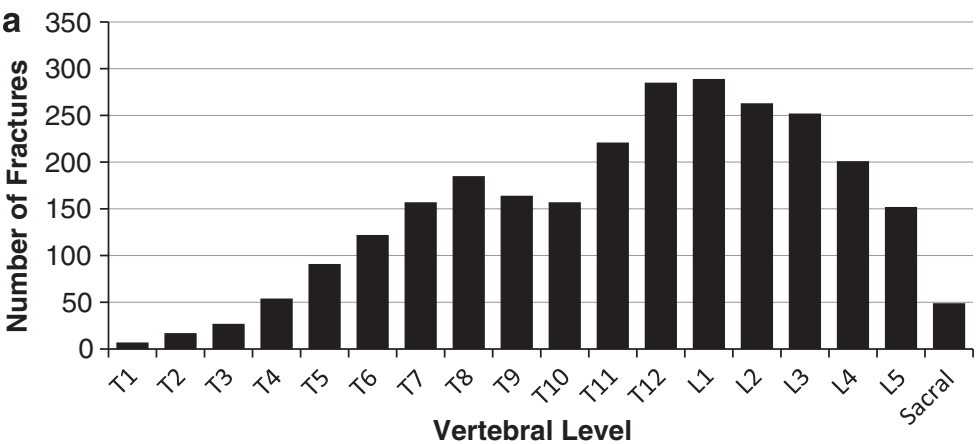

Vertebral Level
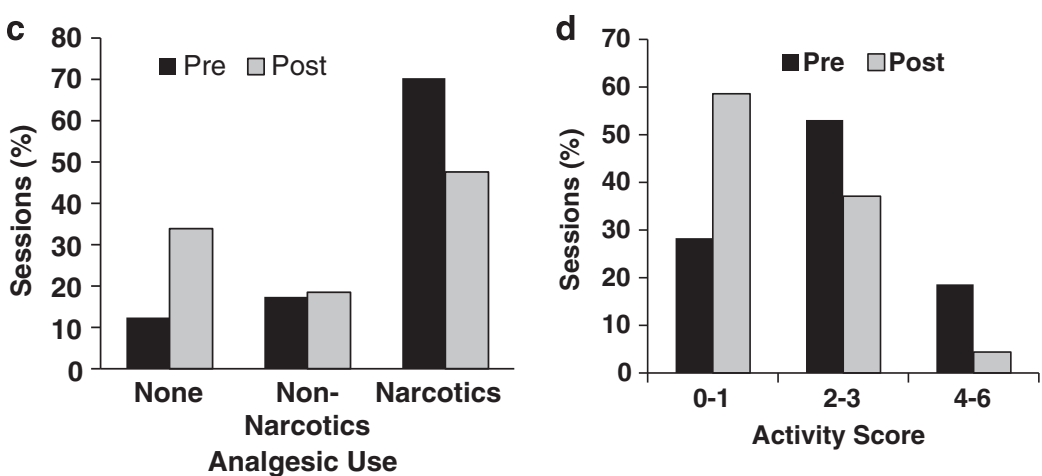
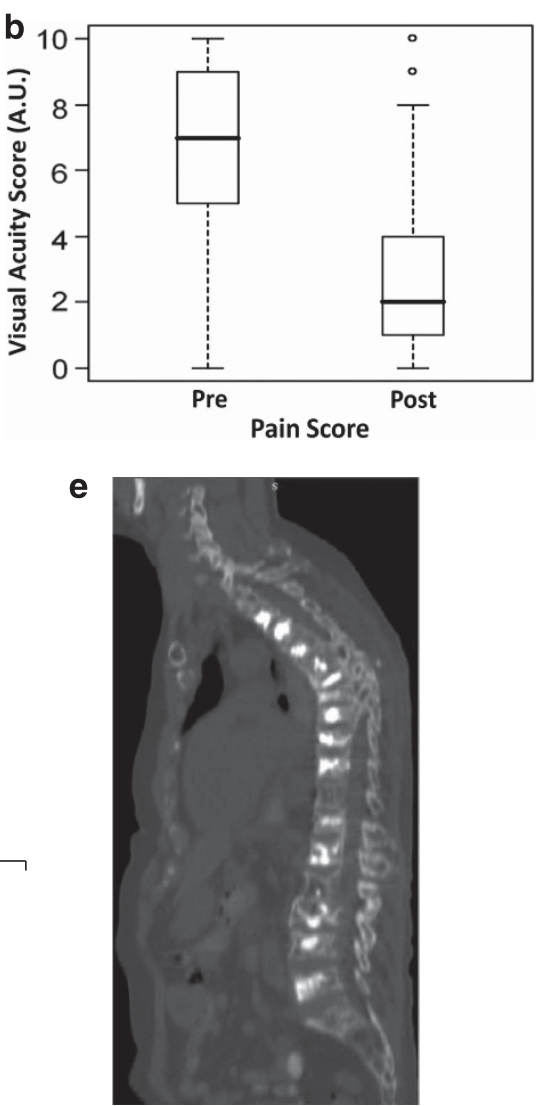

Figure 1. (a) Number of fractures by vertebral level. (b) Reported Visual Acuity Scores (VAS) pain levels pre- and 1-month post-procedure $(n=428$ sessions in 351 patients). Box and whisker plots of pain scores by patient survey indicate levels of VAS pain. (c) Reported analgesics type at preand 1-month post-procedure ( $n=437$ sessions in 355 patients). Analgesic types included either none, non-narcotic or over the counter, or narcotics reported in percent of sessions by patients in pre- (black bar) and 1-month post-procedure (gray bar). Vertebral augmentation showed improvements by increased incidence of the none response and decreased incidence in the narcotics category. Analgesics were reported as percentages (\%) from $n=437$ sessions. (d) Reported patient activity levels at pre- and 1-month post-procedure ( $n=430$ sessions in 354 patients). Patient activity levels were determined by the following: $0=$ no limitation, $1=$ walking without assistance, $2=$ walk needing frequent intervals of rest, $3=$ use of cane or walker, $4=$ use of wheelchair, $5=$ upright in chair or bed, $6=$ flat on bed. Patients reported increased '0-1' activity and decreased limitations at ' $2-3$ ' and ' $4-6$ ' activity scores following vertebral augmentation. Activities were reported as percentages (\%) from $n=430$ sessions. (e) CT (lateral) image of multiple vertebral augmentation repair. Image of repaired compression fractures treated with both vertebroplasty and balloon kyphoplasty in three sessions and 16 consecutive levels from T2 to L5 vertebra.

Reduction in pain

Using the 0-10 VAS scale, ${ }^{9} 351$ subjects provided pre- and 1-month post-procedure assessments of their pain for 428 sessions (Figure 1b). From a baseline pain assessment score of 6.9 to a post score of 2.7, there was an average reduction of 4.2 points $(95 \%$ confidence interval $(\mathrm{Cl}) 4.0-4.5)$ that was significant $(P<0.001)$.

\section{Reduction in analgesic medication}

Pain medication usage pre- and post-procedure was available for 437 sessions in $n=355$ patients. Across all sessions, $12 \%$ of sessions had subjects reporting zero pain medications preprocedure as compared with $34 \%$ post-procedure. Patients were taking narcotics for $70 \%$ of sessions pre-procedure compared to $48 \%$ post-procedure (Figure 1c). Accounting for clustering of sessions within participants, the odds ratio (OR) of narcotics usage was $65 \%$ lower $(\mathrm{OR}=0.35 ; 95 \% \mathrm{Cl}, 0.21-0.58)$ at 1 -month postprocedure as compared with pre-procedure $(P<0.001)$.

Improvement in activity

Activity assessments were provided by 354 subjects across 430 sessions. Across sessions, pre-procedure $28 \%$ of subjects scored 0-1 (that is, no limitations or walking without assistance) as compared to $59 \%$ post-procedure (Figure 1d). Accounting for clustering, the odds of good activity (score of $0-1$ ) was 4.2 $(95 \% \mathrm{Cl}, 3.1-5.8)$ times higher post-procedure as compared to pre-procedure $(P<0.001)$.

There were no significant differences in improvements between the type of procedure performed (kyphoplasty vs vertebroplasty or kyphoplasty + vertebroplasty) for pain relief, decreased narcotics usage or improvement in activity (all $P>0.05$ ), after adjusting for age, gender, session, number of augmentations and baseline scores or medication. The smaller sample sizes should be kept in mind of this comparison, as $74 \%$ of sessions with outcome data involved vertebroplasty only, 13\% kyphoplasty only and 13\% both procedures. For the subset with repairs confined to a single region (T1-T10, T11-L2 and L3-sacrum), there was no difference detected in pain reduction, medication usage and activity according to region (all $P>0.05, n=197-203$ patients).

Our analyses found that vertebral augmentation (vertebroplasty or kyphoplasty) therapy significantly decreased the VAS level of pain, decreased narcotic usage and increased activity levels in multiple myeloma patients with multiple and single repairs (Figure $1 \mathrm{~b}-\mathrm{d})$. Only two $(0.3 \%)$ patients required antibiotics for local infections, and no neurological deficits were observed. From a baseline VAS of 6.9, average pain scores decreased to 2.7 (Figure $1 \mathrm{~b}$ ) following vertebral augmentation. Other recent studies have noted in cancer and osteoporotic patients that vertebral 
Table 1. Distribution of compression fractures (CF) among both types of vertebral augmentation, vertebroplasty and kyphoplasty and number of augmentations per session for $n=792$ subjects

\begin{tabular}{lc}
\hline Procedure/CF vertebrae & $\mathrm{N}(\%)^{\mathrm{a}}$ CF treated \\
\hline Vertebroplasty $(\mathrm{n}=2223)$ & \\
T1-T10 & $816(37)$ \\
T11-L2 & $871(39)$ \\
L3-acrum & $536(24)$ \\
Kyphoplasty $(\mathrm{n}=470)$ & \\
T1-T10 & $165(35)$ \\
T11-L2 & $187(40)$ \\
L3-sacrum & $118(25)$ \\
Total repairs & 2693 \\
\hline Number of augmentations/session & $\mathrm{N}(\%)$ sessions \\
\hline 1 & $337(32)$ \\
2 & $258(24)$ \\
3 & $227(21)$ \\
4 & $134(13)$ \\
5 & $66(6)$ \\
$6-9$ & $47(4)$ \\
Total sessions & 1072 \\
\hline aPercentages based on totals for the respective procedure. \\
\hline
\end{tabular}

augmentation provided significant pain relief and minimal complications. ${ }^{5}$ Myeloma patients who are severely limited by pain to only reclining or sitting ideally benefit from vertebral augmentation. ${ }^{8}$

Multiple augmentation procedures or performing multiple levels at one session may be a necessary and appropriate resolution for pain. Hussein et al. ${ }^{8}$ state that repair of three to four vertebrae per augmentation is reasonable. We also reported up to 16 repairs in one patient in the same paper (Figure 1e). While the majority (75\%) of the 792 patients in this study required a single vertebral augmentation session, the median number of repairs was consistent with other studies at two per session. Augmentation repairs have been noted in levels up to the cervical area, and percutaneous repairs in the T3-L5 levels are considered safe and effective. ${ }^{5,8}$ Others have differed in size requirements to successfully augment vertebral collapse and placed upper limits from T7 to T3. ${ }^{10,11}$ However, repairs in our study were successfully made at the upper T1-T5 thoracic levels $(4.7 \%)$. Subsequent to this study, we have successfully included some cervical levels. Limitations of our study include not assessing the potentially confounding effects of multiple myeloma therapy (for example, steroids) on outcomes, observational design, and participation and recall bias. Outcomes assessment was complicated by cancer therapy and/or relocation, and mortality, which was not assessed in this study, cannot be ruled out as a reason for nonresponse in some cases. This report comprises the largest study of distribution of compression fractures in consecutive multiple myeloma patients and prospectively acquired outcomes in patients undergoing vertebral augmentation to date.

\section{CONFLICT OF INTEREST}

The authors declare no conflict of interest.

E Erdem ${ }^{1}$, R Samant ${ }^{1}$, SF Malak ${ }^{1}$, WC Culp ${ }^{1}$, A Brown ${ }^{1}$, L Peterson ${ }^{1}$, $S$ Lensing ${ }^{2}$ and $B$ Barlogie ${ }^{3}$

${ }^{1}$ Department of Radiology, University of Arkansas for Medical Sciences, Little Rock, AR, USA;

${ }^{2}$ Department of Biostatistics, University of Arkansas for Medical Sciences, Little Rock, AR, USA and

${ }^{3}$ Myeloma Institute for Research and Therapy, University of Arkansas for Medical Sciences, Little Rock, AR, USA E-mail: erdemeren@uams.edu

\section{REFERENCES}

1 American Cancer Society. Cancer Facts and Figures 2012 Atlanta, GA: American Cancer Society, 2012.

2 Morgan GJ, Wu P. Targeting bone in myeloma. Recent Res Cancer Res 2012; 192: 127-143.

3 Giuliani N, Ferretti M, Bolzoni M, Storti P, Lazzaretti M, Dalla Palma B et al. Increased osteocyte death in multiple myeloma patients: role in myelomainduced osteoclast formation. Leukemia 2012; 26: 1391-1401.

4 Ramos L, de Las Heras JA, Sanchez S, González-Porras JR, González R, Mateos MV et al. Medium-term results of percutaneous vertebroplasty in multiple myeloma. Eur J Haematol 2006; 77: 7-13.

5 Burton AW, Mendoza T, Gebhardt R, Hamid B, Nouri K, Perez-Toro M et al. Vertebral compression fracture treatment with vertebroplasty and kyphoplasty: experience in 407 patients with 1,156 fractures in a tertiary cancer center. Pain Med 2011; 12: 1750-1757.

6 Cotton A, Dewatre F, Cortet B, Assaker R, Leblond D, Duquesnoy B et al. Percutaneous vertebroplasty for osteolytic metastases and myeloma: effects of the percentage of lesion filling and the leakage of methyl methacrylate at clinical follow-up. Radiology 1996; 200: 525-530.

7 Lunt M, O'Neill TW, Felsenberg D, Reeve J, Kanis JA, Cooper C et al. Characteristics of a prevalent vertebral deformity predict subsequent vertebral fracture: results from the European Prospective Osteoporosis Study (EPOS). Bone 2003; 33: 505-513.

8 Hussein MA, Vrionis FD, Allison R, Berenson J, Berven S, Erdem E et al. on behalf of the International Myeloma Working Group. The role of vertebral augmentation in multiple myeloma: International myeloma working group consensus statement. Leukemia 2008; 22: 1479-1484.

9 Gerszten PC, Welch WC. Combined percutaneous transpedicular tumor debulking and kyphoplasty for pathological compression fractures. Technical note. J Neurosurg Spine 2007; 6: 92-95.

10 La Maida GA, Sala F, Callea G, Capitani D, Singh S. Efficacy of unipedicular balloon kyphoplasty for treatment of multiple myeloma vertebral lesions. Asian Spine J 2011; 5: 162-168.

11 Hertlein H, Hartl WH, Dienemann H, Schürmann M, Lob G. Thoracoscopic repair of thoracic spine trauma. Eur Spine J 1995; 4: 302-307.

(c) (i) $\Theta$ This work is licensed under a Creative Commons AttributionNonCommercial-NoDerivs 3.0 Unported License. To view a copy of this license, visit http://creativecommons.org/licenses/by-nc-nd/3.0/

\section{Prognostic impact and landscape of NOTCH1 mutations in chronic lymphocytic leukemia (CLL): a study on 852 patients}

\section{Leukemia (2013) 27, 2393-2396; doi:10.1038/leu.2013.218}

Chronic lymphocytic leukemia (CLL) is the most common leukemia in adults. Recently, recurrent activating mutations of NOTCH1 have been reported in up to $12 \%$ of CLL patients, underlining the relevance of $\mathrm{NOTCH} 1$ mutations (NOTCH1mut) as an independent negative prognostic marker. ${ }^{1-3}$ NOTCH1mut in CLL are known to be located predominantly within the C-terminal PEST domain, frequently resulting in a truncated protein that is more stable than the wild-type one and activates the NOTCH1 signaling pathway. $^{3}$ 